\title{
A Note On Quantum Compactons
}

\author{
D. Bazeia ${ }^{1}$ and D.V. Vassilevich ${ }^{2}$ \\ ${ }^{1}$ Departamento de Física Universidade Federal da Paraíba, 58051-900 João Pessoa PB, Brazil \\ ${ }^{2}$ Centro de Matemática, Computação e Cognição, \\ Universidade Federal do ABC, 09210-170 Santo André, SP, Brazil
}

\begin{abstract}
Compactons are solutions of the equations of motion that behave trivially outside a compact region. In general, the operators describing quantum fluctuations above compactons have singularities. However, we show that despite these singularities the quantum theory is well defined. As an example, we calculate the one-loop mass shift of a compacton in a model described by a single real scalar field.
\end{abstract}

PACS numbers: 11.10. Lm, 11.27. +d

\section{INTRODUCTION}

Topological defects appear in nature in a diversity of contexts. In particular, they are of current interest in high energy physics, where they can be used to describe phase transitions in the early universe or map interfaces separating distinct regions in space [1-3]. An interesting recent example of the use of topological defect is the study of magnetic domain wall in a nanowire, intended for the development of magnetic memory at the nanometric scale [4]. Other investigations concerning topological defects in high energy physics can be found for instance in [5].

In this work we study the presence of topological defects in relativistic models described by a real scalar field $\phi$ in $(1+1)$ spacetime dimensions. We focus attention on k-field models, with the kinematics modified to allow for higher order power in the derivative of the dynamical field. These models were introduced to contribute to understand the current accelerated expansion of the Universe [6], and they were also studied with other motivations in Ref. 7-10], for instance. See also [1] for supersymmetric extensions of these models.

Our main focus is on the compacton solutions in models engendering generalized kinematics [8 [10]. We remind that compactons appear in the presence of nonlinearity and nonlinear dispersion, acquiring spatial profile with compact support [12]. They have been investigated in different contexts in [13, 14] and they are spacelike structures similar to kinks; see, e.g. [15] for a recent study on compactons.

Compactons behave trivially outside a compact region. Consequently, the derivatives may develop discontinuities, and the fluctuation operators may have singularities reminding confining domain walls. We study whether despite their exotic properties these solutions can be quantized. We find that the answer is positive and calculate the one-loop shift of the compacton energy.

We organize the work as follows: in Sec. II we briefly review the compacton classical solutions in a model with a single real scalar field in $(1+1)$ spacetime dimensions. In Sec. III we consider the quantum fluctuations and the radiative corrections. We end the work with some com- ments and conclusions in Sec. IV.

\section{CLASSICAL SOLUTIONS}

We consider the models described by the Lagrange density

$$
\mathcal{L}=F(X)-V(\phi)
$$

where $V(\phi)$ is a potential depending on a real scalar field $\phi$. We work in $(1+1)$ spacetime dimensions, with $x^{\mu}=$ $\left(x^{0}=t, x^{1}=x\right), x_{\mu}=\left(x_{0}=t, x_{1}=-x\right)$ and we consider $t, x$ and the field $\phi$ dimensionless, for simplicity. The function $F(X)$ is in principle an arbitrary function of $X$, which is defined as

$$
X=\frac{1}{2} \partial_{\mu} \phi \partial^{\mu} \phi
$$

The most famous model from this class with

$$
\mathcal{L}_{0}=X-\frac{1}{2}\left(1-\phi^{2}\right)^{2}
$$

is the $\phi^{4}$ model, engendering spontaneous symmetry breaking. It supports defect structures, of the kinklike type, given explicitly by

$$
\phi_{0}(x)=\tanh (x)
$$

Less standard models can be described by, for instance,

$$
\mathcal{L}_{n}=-X^{2}-\frac{3}{4}\left(1-\phi^{2}\right)^{2 n}
$$

where $n$ can be 1 or 2 , leading to the models

$$
\begin{aligned}
& \mathcal{L}_{1}=-X^{2}-\frac{3}{4}\left(1-\phi^{2}\right)^{2} \\
& \mathcal{L}_{2}=-X^{2}-\frac{3}{4}\left(1-\phi^{2}\right)^{4} .
\end{aligned}
$$

The equation of motion has the general form

$$
\partial_{\mu}\left(\mathcal{L}_{X} \partial^{\mu} \phi\right)+\frac{d V}{d \phi} .
$$


Here we get

$$
\partial_{\mu} \phi \partial^{\mu} \phi \partial_{\nu} \partial^{\nu} \phi+2 \partial_{\mu} \phi \partial_{\nu} \phi \partial^{\nu} \partial^{\mu} \phi=\frac{d V}{d \phi} .
$$

In this case, for static solution the equations of motion are, for $n=1$

$$
\phi^{\prime 2} \phi^{\prime \prime}=-\phi\left(1-\phi^{2}\right)
$$

and for $n=2$

$$
\phi^{\prime 2} \phi^{\prime \prime}=-2 \phi\left(1-\phi^{2}\right)^{3} .
$$

Also, the static solutions have energy density and stress given by

$$
\begin{aligned}
\rho_{n}(x) & =\frac{1}{4} \phi^{\prime 4}+V_{n}(\phi) ; \\
\tau_{n}(x) & =\frac{3}{4} \phi^{\prime 4}-V_{n}(\phi) .
\end{aligned}
$$

The two models also support defect structures. For the model described by $\mathcal{L}_{1}$ we get

$$
\phi_{1}(x)=\sin (x), \quad-\frac{\pi}{2} \leq x \leq \frac{\pi}{2},
$$

and $\phi_{1}(x)=-1$ for $x \leq-\pi / 2 ; \phi_{1}(x)=1$ for $x \geq \pi / 2$. The energy density is

$$
\rho_{1}(x)=\cos ^{4}(x),
$$

for $x \in[-\pi / 2, \pi / 2] ; \rho_{1}(x)$ vanishes outside this compact region. The energy becomes $E_{1}=3 \pi / 8$. The solution is of the compact type, since it is defined in the real line, but it only deviates from the minima in the compact set $x \in[-\pi / 2, \pi / 2]$. We note that $\phi_{1}(x)=\sin (x)$ is stressless, leading to the first-order equation

$$
\phi^{\prime}=\sqrt{1-\phi^{2}}=W_{1 \phi}^{1 / 3},
$$

where

$$
W_{1}(\phi)=\phi \sqrt{1-\phi^{2}}\left(\frac{5}{8}-\frac{1}{4} \phi^{2}\right)+\frac{3}{8} \arcsin (\phi)
$$

and $W_{1 \phi}=d W_{1} / d \phi$.

For the second model, described by $\mathcal{L}_{2}$, we get

$$
\phi_{2}(x)=\tanh (x) .
$$

This is the same solution we found in the standard $\phi^{4}$ model. See [15] for further details on compactons.

\section{FLUCTUATIONS AND RADIATIVE CORRECTIONS}

Let us study quantum fluctuation above the compacton solution (12) in the model described by $\mathcal{L}_{1}$; see
Eq. (6a). Outside the region where this solution is localized the dynamics of fluctuations is governed by the same action (6a) with a shifted potential,

$$
\begin{array}{ll}
\mathcal{L}(\eta) & =-X^{2}(\eta)-\frac{3}{4}\left(-\eta^{2}+2 \eta\right)^{2}, \quad x<-\pi / 2 \\
\mathcal{L}(\eta) & =-X^{2}(\eta)-\frac{3}{4}\left(\eta^{2}+2 \eta\right)^{2}, \quad x>\pi / 2
\end{array}
$$

Kinetic terms in both actions above are 4th order in fluctuations. Consequently, there are no propagating perturbations outside the compacton. This effect was already noted in 9 .

Inside the region, $-\pi / 2<x<\pi / 2$, the linearized equations of motion lead to the following Schrödingerlike equation

$$
L u_{\omega} \equiv\left(-\frac{d^{2}}{d z^{2}}+U_{1}(z)\right) u_{\omega}(z)=\omega^{2} u_{\omega}(z),
$$

where we have changed $x \rightarrow z$ and $\eta \rightarrow u$, according to

$$
z=3^{1 / 2} x, \quad u_{\omega}=3^{1 / 4} \phi^{\prime} \eta_{\omega} .
$$

$U_{1}(z)$ is the Pöschl-Teller potential 16 .

$$
U_{1}(z)=-12+6 \sec ^{2}(\sqrt{3} z) .
$$

Solutions of the equation (19) are very well known, see 16, 17]:

$$
\begin{aligned}
& u_{2 k}(z)=\cos ^{2}(\sqrt{3} z){ }_{2} F_{1}\left(2+k,-k, \frac{1}{2}, \sin ^{2}(\sqrt{3} z)\right), \\
& \begin{aligned}
u_{2 k+1}(z)=\cos ^{2}(\sqrt{3} z) \sin (\sqrt{3} z) \\
\quad \times{ }_{2} F_{1}\left(3+k,-k, \frac{3}{2}, \sin ^{2}(\sqrt{3} z)\right),
\end{aligned}
\end{aligned}
$$

$k=0,1,2, \ldots$ The hypergeometric functions in the equations above are actually polynomials of $\sin ^{2}(\sqrt{3} z)$ of degree $k$. The corresponding eigenfrequencies read

$$
\omega_{n}=\sqrt{3\left(n^{2}+4 n\right)} .
$$

By looking at the functions (22) we can make an important observation. They all vanish at least as $\cos ^{2}(\sqrt{3} z)$ at the endpoints of the interval $[-\pi / 2 \sqrt{3}, \pi / 2 \sqrt{3}]$. Therefore, the original perturbations $\eta$, see (20), also vanish at these points. This implies that quantum fluctuations cannot escape the region where the compacton is localized.

As an example of quantum computations with the compacton let us calculate the energy of confined fluctuations. Formally, it is given by a half sum of the eigenfrequencies (23)

$$
\mathcal{E}=\frac{1}{2} \sum_{n=1}^{\infty} \omega_{n}
$$

This sum is obviously divergent and has to be regularized and renormalized. We use the zeta-function regularization. Earlier, the heat kernel methods and the zeta 
regularization were applied to calculations of the quantum energy of solitons in [20, 21]. Namely, we introduce a complex parameter $s$ and replace (24)) by

$$
\mathcal{E}_{s}=\frac{1}{2} \sum_{n=1}^{\infty}\left(\omega_{n}^{2}\right)^{\frac{1}{2}-s} .
$$

For $\Re(s)>1$ the sum above converges, but gets divergent contributions (poles) when continued to the physical value $s=0$. To analyze these divergences, it is convenient to make the Mellin transformation

$$
\mathcal{E}_{s}=\frac{1}{\Gamma\left(s-\frac{1}{2}\right)} \int_{0}^{\infty} \frac{d t}{t} t^{s-\frac{1}{2}} K(t),
$$

where

$$
K(t)=\sum_{n=1}^{\infty} e^{-t \omega_{n}^{2}}=\operatorname{Tr}\left(e^{-t L}\right),
$$

is the heat kernel of the second-order differential operator $L$ defined in (19). It is well known, that for $\tau \rightarrow+0$,

$$
\sum_{k=-\infty}^{\infty} e^{-\tau k^{2}} \simeq \sqrt{\frac{\pi}{\tau}}
$$

up to exponentially small terms. By using this formula, one can easily derive the small $t$ asymptotic expansion of the heat kernel (27):

$$
\begin{aligned}
K(t) & \simeq \frac{1}{2} \sqrt{\frac{\pi}{3 t}}-\frac{5}{2}+2 \sqrt{3 \pi t}+O(t), \\
& =a_{0} t^{-1 / 2}+a_{1}+a_{2} t^{1 / 2}+O(t) .
\end{aligned}
$$

As discussed in [18], the heat kernel coefficients $a_{0}, a_{1}$ and $a_{2}$ describe one-loop divergences of the vacuum energy in two dimensions in various regularization schemes. $a_{0}$ corresponds to quadratic divergences, $a_{1}$ - to linear divergences, and $a_{2}$ - to logarithmic ones. In a sense, the heat kernel coefficients tell which "effective geometry" is seen by the quantum fluctuations. For a non-singular potential, there are analytic expressions for these coefficients, see e.g. [19]. In particular, the $a_{0}$ coefficient has to be $(4 \pi)^{-1 / 2}$ times the length of the interval, $\pi / \sqrt{3}$. This value is consistent with (29). The $a_{1}$ coefficient appears if there are boundaries. For a smooth potential on an interval with Dirichlet boundary conditions at both endpoints it has to be $-1 / 2$, which differs from the value in (29). In the nonsingular case $a_{2}$ is proportional to the integral of the potential. In our case this integral is divergent, so that no comparison is possible. Generally speaking, there is no theory of the heat kernel expansion for singular potentials like (21). As well, there are no general methods of renormalization. Developing such methods is an interesting problem, which we shall not address here. We shall use the minimal subtraction scheme, that does not require precise understanding of the counterterms.

In the zeta-function regularization only the logarithmic divergence shows up, that becomes a pole $1 / s$. By using
(26) one can easily show that the pole contribution to the vacuum energy reads [18]

$$
\mathcal{E}_{\text {pole }}=-\frac{a_{2}}{4 \sqrt{\pi}} \frac{1}{s}
$$

Let us now represent the sum (25) as an integral in the complex plane

$$
\mathcal{E}_{s}=\oint \frac{d y}{4 i}\left(3\left(y^{2}+4 y\right)\right)^{\frac{1}{2}-s} \operatorname{ctg}(\pi y),
$$

where the contour goes anti-clockwise around the poles of $\operatorname{ctg}(\pi y)$ at the points $y=1,2,3, \ldots$ Next, we rotate the upper part of the contour to the vertical line $y=\frac{1}{2}+i w$, and the lower part - to $y=\frac{1}{2}-i w, w \geq 0$; we get

$$
\begin{gathered}
\mathcal{E}_{s}=-\frac{1}{4} \int_{0}^{\infty} d w \tanh (\pi w)\left(e^{-i \pi s}\left[3\left(w^{2}-\frac{9}{4}-5 i w\right)\right]^{\frac{1}{2}-s}\right. \\
\left.+e^{i \pi s}\left[3\left(w^{2}-\frac{9}{4}+5 i w\right)\right]^{\frac{1}{2}-s}\right) .
\end{gathered}
$$

To evaluate this integral, we represent $\tanh (\pi w)=1+$ $(\tanh (\pi w)-1)$. The integral of the first (constant) term is divergent at $s=0$. It has to be evaluated analytically, yielding a pole $1 / s$ and a finite contribution. The integral of $(\tanh (\pi w)-1)$ is finite at $s=0$, and we can put $s=0$ there immediately. After the calculations, we have for $s \sim 0$ :

$$
\mathcal{E}_{s} \simeq-\frac{\sqrt{3}}{2 s}+2.29822 \ldots
$$

The pole part of this expression is consistent with (30). As announced above, we use the minimal subtraction scheme. Namely, we subtract from $\mathcal{E}_{s}$ the pole (30) and nothing more. The renormalized value for the vacuum energy thus becomes

$$
\mathcal{E}_{\text {ren }}=\left(\mathcal{E}_{s}-\mathcal{E}_{\text {pole }}\right)_{s=0}=2.29822 \ldots
$$

Since the spectrum of quantum fluctuations is known explicitly, see (22) and (23), one can immediately construct the propagators and calculate quantum corrections to other quantities of interest.

\section{COMMENTS AND CONCLUSIONS}

In this work we have investigated quantum corrections to the mass of a compacton in one of the k-field models. This quantum correction is well defined. In general, quantum theory on the background of a compacton does not look more complicated than the one above regular solitons. A complete renormalization theory is still missing, but this is rather a feature of all k-field models than a specific drawback of compactons.

In conclusion, compactons provide a natural mechanism of confinement and can be successfully quantized. They definitely deserve more attention. 
As a particularly interesting route, we recall that in the recent work [22] one shows how to get from kinks to compactons in models with standard kinematics. This route seems to provide an alternative way to investigate how the quantum corrections behave in the limit where the kinklike solution tends to become a compacton.

\section{Acknowledgments}

The authors would like to thank CNPq and FAPESP for partial financial support.
[1] R. Rajaraman, Solitons and Instantons (North-Holland, Amsterdan, 1982).

[2] A. Vilenkin and E. P. S. Shellard, Cosmig Strings and Others Topological Defects (Cambridge, UK, 1994).

[3] N. Manton and P. Sutcliffe, Topological Solitions (Cambridge UP, Cambridge, UP, Cambridge, UK, 2004).

[4] A. Vanhaverbeke, A. Bischof and R. Allenspach, Phys. Rev. Lett. 101, 107202 (2008).

[5] G. Basar and G.V. Dunne, Phys. Rev. Lett. 100, 200404 (2008); S. Dutta, D. A. Steer, and T. Vachaspati, Phys. Rev. Lett. 101, 121601 (2008); A. Alonso-Izquierdo, M.A. Gonzalez Leon, and J. Mateos Guilarte, Phys. Rev. Lett. 101, 131602 (2008); T. Romanczukiewicz and Ya. Shnir, Phys. Rev. Lett. 105, 081601 (2010); P. Dorey, K. Mersh, T. Romanczukiewicz, and Ya. Shnir, Phys. Rev. Lett. 107, 091602 (2011).

[6] C. Armendariz-Picon, T. Damour and V. F. Mukhanov, Phys. Lett. B 458209 (1999); T. Chiba, T. Okabe and M. Yamaguchi, Phys. Rev. D 62, 023511 (2000); C. Armendariz-Picon, V. F. Mukhanov and P. J. Steinhardt, Phys. Rev. Lett. 85, 4438 (2000).

[7] E. Babichev, Phys. Rev. D 74, 085004 (2006); D. Bazeia, L. Losano, R. Menezes and J.C.R. Oliveira, Eur. Phys. J. C 51, 953 (2007); E. Babichev, Phys. Rev. D 77, 065021 (2008).

[8] C. Adam, J. Sanchez-Guillen and A. Wereszczynski, J. Phys. A 40, 13625 (2007).

[9] C. Adam, N. Grandi, J. Sanchez-Guillen and A. Wereszczynski, J. Phys. A 41, 212004 (2008) [Erratum-ibid. A 42, 159801 (2009)].

[10] D. Bazeia, L. Losano, R. Menezes, Phys. Lett. B 668, 246 (2008).

[11] D. Bazeia, R. Menezes, A.Yu. Petrov, Phys. Lett. B 683,
335 (2010); C. Adam, J.M. Queiruga, J. Sanchez Guillen, A. Wereszynski, Phys. Rev. D 84, 065032 (2011).

[12] P. Rosenau and J.M. Hyman, Phys. Rev. Lett. 70, 564 (1993).

[13] P. Rosenau, Phys. Rev. Lett. 73, 1737 (1994); P. Rosenau and A. Pikovsky, Phys. Rev. Lett. 94, 174102 (2005); P. Rosenau, J.M. Hyman, and M. Staley, Phys. Rev. Lett. 98, 024101 (2007); P. Rosenau and E. Kashdan, Phys. Rev. Lett. 101, 264101 (2008); P. Rosenau and E. Kashdan, Phys. Rev. Lett. 104, 034101 (2010); F.Kh. Abdullaev, P.G. Kevrekidis, and M. Salerno, Phys. Rev. Lett. 105, 113901 (2010).

[14] F. Cooper, H. Sheppard, and P. Sodano, Phys. Rev. E 48, 4027 (1993); A. Khare and F. Cooper, Phys. Rev. E 48, 4843 (1993); F. Cooper, J.M. Hyman, and A. Khare, Phys. Rev. E 64, 026608 (2001).

[15] D. Bazeia, L. Losano, and R. Menezes, Phys. Lett. B 731, 293 (2014).

[16] G. Pöschl and E. Teller, Z. Phys. 83, 143 (1933); N. Rosen and P.M. Morse, Phys. Rev. 42, 210 (1932).

[17] S. Flügge, Rechenmetoden der Quantentheorie (Springer, Berlin, 1965).

[18] D.V. Fursaev and D.V. Vassilevich, Operators, Geometry and Quanta. Methods of spectral geometry in quantum field theory (Springer, Dordrecht, 2011).

[19] D.V. Vassilevich, Phys. Rept. 388, 279 (2003).

[20] M. Bordag, J. Phys. A 28, 755 (1995).

[21] M. Bordag, A.S. Goldhaber, P. van Nieuwenhuizen, and D. Vassilevich, Phys. Rev. D 66, 125014 (2002).

[22] D. Bazeia, L. Losano, M.A. Marques, and R. Menezes, Phys. Lett. B 736, 515 (2014). 